\title{
PAIRS OF CONSECUTIVE PRIMITIVE ROOTS MODULO A PRIME
}

\author{
EMANUEL VEGH
}

A. Brauer [1] has shown that given an integer $N$, there are at least $N$ consecutive quadratic nonresidues modulo $p$ for all sufficiently large primes $p$. A similar result, if true, for the primitive roots modulo $p$ appears to be difficult to prove. A. Brauer has asked (oral communication) if, for all sufficiently large primes, there is a pair of consecutive primitive roots. In this direction we prove the

TheOREM. If $p$ is a prime greater than 3 such that $\Phi(p-1) /(p-1)$ $>1 / 3$, where $\Phi$ is the Euler totient function, then $p$ has consecutive primitive roots.

First, we prove the

Leммa. If $p$ is a prime greater than 3 then exactly half the primitive roots modulo $p$ are followed by quadratic nonresidues.

Proof of the Lemma. Let $\xi$ be a primitive root modulo $p$. Now $p-1$ and $p-2$ are relatively prime and therefore $\xi^{p-2}$ is also a primitive root modulo $p$. Furthermore $\xi \not \xi^{p-2} \bmod p$ since $p \neq 3$. Since $\xi$ is also a quadratic nonresidue, it follows from the congruence

$$
\xi\left(\xi^{p-2}+1\right)=\xi^{p-1}+\xi \equiv \xi+1 \quad \text { modulo } p
$$

that $\xi+1$ is a quadratic nonresidue if and only if $\xi^{p-2}+1$ is a quadratic residue. Thus exactly one of the primitive roots $\xi$ and $\xi^{p-2}$ is followed by a quadratic nonresidue. It is easy to see that if $\eta$ is a primitive root incongruent to $\xi$ and $\xi^{p-2}$ then $\eta^{p-2}$ is also incongruent to $\xi$ and $\xi^{p-2}$. The lemma then follows by considering all pairs of primitive roots $\xi$ and $\xi^{p-2}$.

Proof of The Theorem. Let $p$ be a prime greater than 3 such that $\Phi(p-1) /(p-1)>1 / 3$. If we assume that there are no consecutive primitive roots modulo $p$, then using the Lemma, one half of the $\Phi(p-1)$ primitive roots are followed by quadratic nonresidues, no one of which is a primitive root. Since each of the primitive roots is a quadratic nonresidue it follows that there are then at least $(3 / 2) \Phi(p-1)$ quadratic nonresidues. Since there is a total $(p-1) / 2$ quadratic nonresidues, $(3 / 2) \Phi(p-1) \leqq(p-1) / 2$, a contradiction. The theorem is thus proved.

Received by the editors June 13, 1967. 
It might be noted that, as a consequence of the theorem cited in [1], for all sufficiently large primes of the form $2^{n}+1$, there are arbitrarily long sequences of primitive roots, since here each quadratic nonresidue is also a primitive root.

\section{REFERENCE}

1. A. Brauer, Über Sequenzen von Potenzresten, S.-B. Preuss. Akad. Wiss., 1928, pp. 9-16.

Naval Research Laboratory, Washington, D. C. 